\title{
Search for a Higgs Portal Scalar Decaying to Electron-Positron Pairs in the MicroBooNE Detector
}

P. Abratenko, ${ }^{35}$ R. An, ${ }^{15}$ J. Anthony, ${ }^{4}$ J. Asaadi, ${ }^{34}$ A. Ashkenazi, ${ }^{20,32}$ S. Balasubramanian, ${ }^{12}$ B. Baller, ${ }^{12}$ C. Barnes, ${ }^{21}$ G. Barr, ${ }^{25}$ V. Basque, ${ }^{19}$ L. Bathe-Peters,${ }^{14}$ O. Benevides Rodrigues, ${ }^{31}$ S. Berkman, ${ }^{12}$ A. Bhanderi, ${ }^{19}$ A. Bhat,${ }^{31}$ M. Bishai, ${ }^{2}$ A. Blake, ${ }^{17}$ T. Bolton, ${ }^{16}$ J. Y. Book, ${ }^{14}$ L. Camilleri, ${ }^{10}$ D. Caratelli, ${ }^{12}$ I. Caro Terrazas, ${ }^{9}$ R. Castillo Fernandez, ${ }^{12}$ F. Cavanna, ${ }^{12}$ G. Cerati, ${ }^{12}$ Y. Chen, ${ }^{1}$ D. Cianci, ${ }^{10}$ J. M. Conrad,${ }^{20}$ M. Convery, ${ }^{28}$ L. Cooper-Troendle, ${ }^{38}$ J. I. Crespo-Anadón, ${ }^{6}$ M. Del Tutto, ${ }^{12}$ S. R. Dennis, ${ }^{4}$ A. Devitt, ${ }^{17}$ R. Diurba, ${ }^{22}$ R. Dorrill, ${ }^{15}$ K. Duffy, ${ }^{12}$ S. Dytman, ${ }^{26}$ B. Eberly,${ }^{30}$ A. Ereditato, ${ }^{1}$ J. J. Evans,${ }^{19}$ R. Fine, ${ }^{18}$ G. A. Fiorentini Aguirre, ${ }^{29}$ R. S. Fitzpatrick, ${ }^{21}$ B. T. Fleming, ${ }^{38}$ N. Foppiani, ${ }^{14}$ D. Franco, ${ }^{38}$ A. P. Furmanski, ${ }^{22}$ D. Garcia-Gamez, ${ }^{13}$ S. Gardiner, ${ }^{12}$ G. Ge, ${ }^{10}$ S. Gollapinni, ${ }^{33,18}$ O. Goodwin, ${ }^{19}$ E. Gramellini, ${ }^{12}$ P. Green, ${ }^{19}$ H. Greenlee, ${ }^{12}$ W. Gu, ${ }^{2}$ R. Guenette, ${ }^{14}$ P. Guzowski $\odot,{ }^{19}$ L. Hagaman, ${ }^{38}$ E. Hall, ${ }^{20}$ O. Hen, ${ }^{20}$ G. A. Horton-Smith, ${ }^{16}$ A. Hourlier, ${ }^{20}$ R. Itay, ${ }^{28}$ C. James, ${ }^{12}$ X. Ji, ${ }^{2}$ L. Jiang,${ }^{36}$ J. H. Jo, ${ }^{38}$ R. A. Johnson, ${ }^{8}$ Y.-J. Jwa, ${ }^{10}$ N. Kamp,${ }^{20}$ N. Kaneshige, ${ }^{3}$ G. Karagiorgi, ${ }^{10}$ W. Ketchum, ${ }^{12}$ M. Kirby, ${ }^{12}$ T. Kobilarcik, ${ }^{12}$ I. Kreslo, ${ }^{1}$ R. LaZur, ${ }^{9}$ I. Lepetic,${ }^{27}$ K. Li,${ }^{38}$ Y. Li, ${ }^{2}$ K. Lin,${ }^{18}$ B. R. Littlejohn, ${ }^{15}$ W. C. Louis, ${ }^{18}$ X. Luo, ${ }^{3}$ K. Manivannan, ${ }^{31}$ C. Mariani, ${ }^{36}$ D. Marsden, ${ }^{19}$ J. Marshall, ${ }^{37}$ D. A. Martinez Caicedo, ${ }^{29}$ K. Mason, ${ }^{35}$ A. Mastbaum, ${ }^{27}$ N. McConkey, ${ }^{19}$ V. Meddage, ${ }^{16}$ T. Mettler, ${ }^{1}$ K. Miller, ${ }^{7}$ J. Mills,${ }^{35}$ K. Mistry, ${ }^{19}$ A. Mogan, ${ }^{33}$ T. Mohayai, ${ }^{12}$ J. Moon, ${ }^{20}$ M. Mooney, ${ }^{9}$ A. F. Moor, ${ }^{4}$ C. D. Moore, ${ }^{12}$ L. Mora Lepin, ${ }^{19}$ J. Mousseau, ${ }^{21}$ M. Murphy, ${ }^{36}$ D. Naples,${ }^{26}$ A. Navrer-Agasson, ${ }^{19}$ R. K. Neely, ${ }_{16}$ J. Nowak,${ }^{17}$ M. Nunes, ${ }^{31}$ O. Palamara, ${ }^{12}$ V. Paolone, ${ }^{26}$ A. Papadopoulou, ${ }^{20}$ V. Papavassiliou, ${ }^{23}$ S. F. Pate ${ }^{23}$ A. Paudel, ${ }^{16}$ Z. Pavlovic, ${ }^{12}$ E. Piasetzky ${ }^{32}$ I. D. Ponce-Pinto, ${ }^{10,38}$ S. Prince, ${ }^{14}$ X. Qian, ${ }^{2}$ J. L. Raaf,${ }^{12}$ V. Radeka, ${ }^{2}$ A. Rafique, ${ }^{16}$ M. Reggiani-Guzzo, ${ }^{19}$ L. Ren, ${ }^{23}$ L. C. J. Rice, ${ }^{26}$ L. Rochester, ${ }^{28}$ J. Rodriguez Rondon, ${ }^{29}$ H. E. Rogers, ${ }^{5}$ M. Rosenberg, ${ }^{26}$ M. Ross-Lonergan, ${ }^{10}$ G. Scanavini, ${ }^{38}$ D. W. Schmitz, ${ }^{7}$ A. Schukraft, ${ }^{12}$ W. Seligman, ${ }^{10}$ M. H. Shaevitz, ${ }^{10}$ R. Sharankova, ${ }^{35}$ J. Shi, ${ }^{4}$ H. Siegel,${ }^{10}$ J. Sinclair, ${ }^{1}$ A. Smith, ${ }^{4}$ E. L. Snider, ${ }^{12}$ M. Soderberg, ${ }^{31}$ S. Söldner-Rembold ${ }^{19}$ P. Spentzouris, ${ }^{12}$ J. Spitz, ${ }^{21}$ M. Stancari, ${ }^{12}$ J. St. John, ${ }^{12}$ T. Strauss, ${ }^{12}$ K. Sutton, ${ }^{10}$ S. Sword-Fehlberg, ${ }^{23}$ A. M. Szelc, ${ }^{19,11}$ N. Tagg, ${ }^{24}$ W. Tang, ${ }^{33}$ K. Terao,${ }^{28}$ C. Thorpe,${ }^{17}$ D. Totani,${ }^{3}$ M. Toups,${ }^{12}$ Y.-T. Tsai, ${ }^{28}$ M. A. Uchida, ${ }^{4}$ T. Usher, ${ }^{28}$ W. Van De Pontseele, ${ }^{25,14}$ B. Viren, ${ }^{2}$ M. Weber, ${ }^{1}$ H. Wei, ${ }^{2}$ Z. Williams, ${ }^{34}$ S. Wolbers, ${ }^{12}$ T. Wongjirad, ${ }^{35}$ M. Wospakrik, ${ }^{12}$ K. Wresilo, ${ }^{4}$ N. Wright,${ }^{20} \mathrm{~W}$. Wu, ${ }^{12}$ E. Yandel, ${ }^{3}$ T. Yang, ${ }^{12}$ G. Yarbrough, ${ }^{33}$ L. E. Yates, ${ }^{20}$ G. P. Zeller, ${ }^{12}$ J. Zennamo, ${ }^{12}$ and C. Zhang ${ }^{2}$

\section{(MicroBooNE Collaboration)}

\author{
${ }^{1}$ Universität Bern, Bern CH-3012, Switzerland \\ ${ }^{2}$ Brookhaven National Laboratory (BNL), Upton, New York 11973, USA \\ ${ }^{3}$ University of California, Santa Barbara, California 93106, USA \\ ${ }^{4}$ University of Cambridge, Cambridge CB3 OHE, United Kingdom \\ ${ }^{5}$ St. Catherine University, Saint Paul, Minnesota 55105, USA \\ ${ }^{6}$ Centro de Investigaciones Energéticas, Medioambientales y Tecnológicas (CIEMAT), Madrid E-28040, Spain \\ ${ }^{7}$ University of Chicago, Chicago, Illinois 60637, USA \\ ${ }^{8}$ University of Cincinnati, Cincinnati, Ohio 45221, USA \\ ${ }^{9}$ Colorado State University, Fort Collins, Colorado 80523, USA \\ ${ }^{10}$ Columbia University, New York, New York 10027, USA \\ ${ }^{11}$ University of Edinburgh, Edinburgh EH9 3FD, United Kingdom \\ ${ }^{12}$ Fermi National Accelerator Laboratory (FNAL), Batavia, Illinois 60510, USA \\ ${ }^{13}$ Universidad de Granada, Granada E-18071, Spain \\ ${ }^{14}$ Harvard University, Cambridge, Massachusetts 02138, USA \\ ${ }^{15}$ Illinois Institute of Technology (IIT), Chicago, Illinois 60616, USA \\ ${ }^{16}$ Kansas State University (KSU), Manhattan, Kansas 66506, USA \\ ${ }^{17}$ Lancaster University, Lancaster LA1 4YW, United Kingdom \\ ${ }^{18}$ Los Alamos National Laboratory (LANL), Los Alamos, New Mexico 87545, USA \\ ${ }^{19}$ The University of Manchester, Manchester M13 9PL, United Kingdom \\ ${ }^{20}$ Massachusetts Institute of Technology (MIT), Cambridge, Massachusetts 02139, USA \\ ${ }^{21}$ University of Michigan, Ann Arbor, Michigan 48109, USA \\ ${ }^{22}$ University of Minnesota, Minneapolis, Minnesota 55455, USA \\ ${ }^{23}$ New Mexico State University (NMSU), Las Cruces, New Mexico 88003, USA \\ ${ }^{24}$ Otterbein University, Westerville, Ohio 43081, USA
}




\author{
${ }^{25}$ University of Oxford, Oxford OX1 3RH, United Kingdom \\ ${ }^{26}$ University of Pittsburgh, Pittsburgh, Pennsylvania 15260, USA \\ ${ }^{27}$ Rutgers University, Piscataway, New Jersey 08854, USA \\ ${ }^{28}$ SLAC National Accelerator Laboratory, Menlo Park, California 94025, USA \\ ${ }^{29}$ South Dakota School of Mines and Technology (SDSMT), Rapid City, South Dakota 57701, USA \\ ${ }^{30}$ University of Southern Maine, Portland, Maine 04104, USA \\ ${ }^{31}$ Syracuse University, Syracuse, New York 13244, USA \\ ${ }^{32}$ Tel Aviv University, Tel Aviv 69978, Israel \\ ${ }^{33}$ University of Tennessee, Knoxville, Tennessee 37996, USA \\ ${ }^{34}$ University of Texas, Arlington, Texas 76019, USA \\ ${ }^{35}$ Tufts University, Medford, Massachusetts 02155, USA \\ ${ }^{36}$ Center for Neutrino Physics, Virginia Tech, Blacksburg, Virginia 24061, USA \\ ${ }^{37}$ University of Warwick, Coventry CV4 7AL, United Kingdom \\ ${ }^{38}$ Wright Laboratory, Department of Physics, Yale University, New Haven, Connecticut 06520, USA
}

(Received 2 June 2021; revised 11 August 2021; accepted 10 September 2021; published 6 October 2021)

\begin{abstract}
We present a search for the decays of a neutral scalar boson produced by kaons decaying at rest, in the context of the Higgs portal model, using the MicroBooNE detector. We analyze data triggered in time with the Fermilab NuMI neutrino beam spill, with an exposure of $1.93 \times 10^{20}$ protons on target. We look for monoenergetic scalars that come from the direction of the NuMI hadron absorber, at a distance of $100 \mathrm{~m}$ from the detector, and decay to electron-positron pairs. We observe one candidate event, with a standard model background prediction of $1.9 \pm 0.8$. We set an upper limit on the scalar-Higgs mixing angle of $\theta<(3.3-4.6) \times 10^{-4}$ at the $95 \%$ confidence level for scalar boson masses in the range (100-200) $\mathrm{MeV} / c^{2}$. We exclude, at the $95 \%$ confidence level, the remaining model parameters required to explain the central value of a possible excess of $K_{L}^{0} \rightarrow \pi^{0} \nu \bar{\nu}$ decays reported by the KOTO collaboration. We also provide a model-independent limit on a new boson $X$ produced in $K \rightarrow \pi X$ decays and decaying to $e^{+} e^{-}$.
\end{abstract}

DOI: 10.1103/PhysRevLett.127.151803

The Higgs portal model [1] is an extension to the standard model in which an electrically neutral real singlet scalar boson $(S)$ mixes with the Higgs boson with mixing angle $\theta$. Through this mixing, $S$ acquires a coupling to standard model fermions proportional to $\sin \theta$ and their Yukawa couplings with the Higgs boson. For masses between twice the electron mass and twice the muon mass, and assuming that there are no new dark sector particles lighter than half its mass, $S$ will decay to electron-positron pairs with partial width [2]

$$
\Gamma=\theta^{2} \frac{m_{e}^{2} m_{S}}{8 \pi v^{2}}\left(1-\frac{4 m_{e}^{2}}{m_{S}^{2}}\right)^{\frac{3}{2}},
$$

where $m_{S}$ is the scalar boson mass, $m_{e}$ is the electron mass, and $v$ is the Higgs field vacuum expectation value. For these masses, $S$ can be produced from a kaon two-body decay in association with a pion, with the dominant production process being a penguin diagram with a top

Published by the American Physical Society under the terms of the Creative Commons Attribution 4.0 International license. Further distribution of this work must maintain attribution to the author(s) and the published article's title, journal citation, and DOI. Funded by SCOAP ${ }^{3}$. quark running in the loop. The partial width of the production process is [2]

$$
\Gamma \simeq \frac{\theta^{2}}{16 \pi m_{K}}\left|\frac{3 V_{t d}^{*} V_{t s} m_{t}^{2} m_{K}^{2}}{32 \pi^{2} v^{3}}\right|^{2} \lambda^{1 / 2}\left(1, \frac{m_{S}^{2}}{m_{K}^{2}} \frac{m_{\pi}^{2}}{m_{K}^{2}}\right)
$$

where $m_{K}$ is the kaon mass, $m_{\pi}$ is the pion mass, $m_{t}$ is the top quark mass, $V_{t d}$ and $V_{t s}$ are the elements of the Cabibbo-Kobayashi-Maskawa matrix, and $\lambda$ is the Källen Lambda function.

In 2019, the KOTO collaboration reported [3] the observation of four $K_{L}^{0} \rightarrow \pi^{0}+$ invisible decay candidates, a rate 2 orders of magnitude more frequent than the standard model prediction for $K_{L}^{0} \rightarrow \pi^{0} \nu \bar{\nu}$ decays. One candidate was rejected due to upstream veto activity, but three candidates remained as unexplained. In a recent publication [4], they have reevaluated their background expectation to $1.22 \pm 0.26$ counts, and the statistical significance of the observed data has reduced to a $p$ value of 0.13 . The Higgs portal model could explain [5-7] any excess in the KOTO dataset. The value of $\theta$ for a central value of 1.78 counts $\left(\theta_{\mathrm{KCV}}\right)$ is $\approx(4-5) \times 10^{-4}$ over the $m_{S}$ range of $(100-200) \mathrm{MeV} / c^{2}$. The E949 collaboration excludes [8] $\theta_{\mathrm{KCV}}$ for $m_{S}<120 \mathrm{MeV} / c^{2}$, and the NA62 collaboration excludes it [9] for $m_{S}>160 \mathrm{MeV} / c^{2}$. 
This Letter presents the first search for beyond the standard model (BSM) electron-positron pair production in a liquid argon time projection chamber using the MicroBooNE detector, and is the second search for BSM physics in MicroBooNE following a search for heavy neutral leptons [10]. We use the results of this search to exclude, at 95\% confidence level, the remaining Higgs portal model parameter space where $\theta_{\mathrm{KCV}}$ has not been excluded.

The MicroBooNE experiment is primarily designed for neutrino scattering measurements in Fermilab's Booster Neutrino Beam (BNB) [11,12]. The detector sits just below the surface, and comprises an 85 ton liquid-argon time projection chamber (TPC) with active dimensions of $2.6 \mathrm{~m}$ along the drift direction (horizontal and perpendicular to the beam axis; $x$ coordinate in the detector reference frame), $2.3 \mathrm{~m}$ in the vertical direction ( $y$ coordinate), and $10.4 \mathrm{~m}$ along the direction parallel to the BNB direction ( $z$ coordinate). Charged particles traversing the argon produce ionization electrons and scintillation light. Drifted ionization electrons are recorded by three wire planes with $3 \mathrm{~mm}$ pitch that are oriented at $60^{\circ}$ rotations relative to each other. An array of 32 eight-inch photomultiplier tubes (PMTs) distributed behind the wire planes provides timing information for scintillation signals produced inside the cryostat. Part way through the detector operations, a cosmic ray tagger (CRT) system [13] was installed, with four walls of plastic scintillator panels situated along the top, bottom, and long sides of the cryostat that provide timing coincidence signals for some cosmic rays that enter the TPC.

In addition to being on the BNB beam line, the MicroBooNE detector is also situated at a distance of $680 \mathrm{~m}$ and $8^{\circ}$ off axis from the target of Fermilab's Neutrinos at the Main Injector (NuMI) neutrino beam [14], which we have previously used to measure electron neutrino interactions [15]. A schematic diagram of the detector position within the beam line is presented in Fig. 1. The Main Injector delivers $120 \mathrm{GeV}$ protons that impact the graphite target, producing secondary hadrons. A system of electromagnetic horns focuses charged particles either

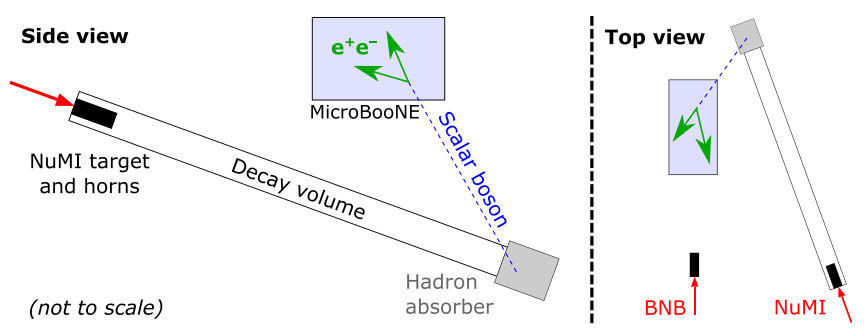

FIG. 1. Schematic of MicroBooNE in the NuMI and BNB beam lines, and the signal signature we are searching for. toward or away from the beam axis, depending on the horn polarity. In forward (reverse) horn current mode, positively (negatively) charged mesons are bent toward the beam axis to produce a beam mostly of neutrinos (antineutrinos) from the meson decays. A $675 \mathrm{~m}$ long helium-filled decay volume is situated downstream of the target and horn system, at the end of which is a $5 \mathrm{~m}$ deep hadron absorber. Any surviving hadrons will be stopped in the absorber, and may produce secondary mesons including $\mathrm{K}^{+}$which will decay at rest. The absorber is at a distance of $\approx 100 \mathrm{~m}$ from the MicroBooNE detector and at an angle of $\approx 125^{\circ}$ with respect to the BNB direction, such that any particles that enter the detector from the absorber are entering in the opposite direction compared to most neutrino interactions seen by the detector. We exploit the unique decay signature of scalar bosons produced by $K^{+}$decaying at rest (KDAR) in the NuMI hadron absorber to search for evidence of the Higgs portal scalar model.

We analyze $0.92 \times 10^{20}$ protons on target (POT) of exposure during run 1 of MicroBooNE's operations (during 2015-2016), and $1.01 \times 10^{20}$ POT of run 3 data (20172018). During the run 1 dataset period, the NuMI beam operated in forward horn current mode, and reverse horn current mode was used during the run 3 period. The CRT had been fully installed by the run 3 period, and we use its information in the analysis of data from that period. The beam-on data is read out from the detector (an "event") when there is a NuMI beam spill timing signal sent by the Fermilab accelerator complex. An on-line trigger is employed to record only those events that pass optical trigger criteria based on the total integrated charge summed over all PMTs in a $100 \mathrm{~ns}$ window. This trigger requires at least one PMT to produce a signal in time with the beam, and the integrated charge has to be above a configurable photoelectron threshold.

To estimate the cosmic-induced backgrounds, we record a dataset of events produced out of time with both beams that employs the same trigger thresholds, called the beamoff dataset. In addition, there is an unbiased dataset of outof-time events for which the trigger is not applied. This unbiased dataset forms the basis of the simulated data. The hit pattern of simulated signal decays or background neutrino interactions are overlaid on top of the unbiased data on an event-by-event level, which allows the cosmic contamination of signal or neutrino interactions to be estimated.

To simulate the scalar boson signal, we use the G4NUMI program [16] which employs a GEANT4 [17] simulation of the NuMI beam line to produce the position and timing distribution of KDAR in the NuMI hadron absorber. For the absolute rate, we use the MiniBooNE estimate [18] of 0.085 muon neutrinos from KDAR in the NuMI hadron 
absorber per POT. The scalars are emitted isotropically from the kaon decay positions, and the scalar's velocity and lab-frame lifetime is used to distribute the scalar decay position, keeping only those that decay within the detector active volume. The electron-positron pair is simulated isotropically in the rest frame, and boosted by the scalar's momentum.

The G4NUMI program is also used to simulate the flux of neutrinos that intersect the detector, which produce the other component of the background to this search. We use the same PPFX [16,19] package as used by the MINERvA [20] and NOvA [21] collaborations to correct the central value neutrino flux prediction and provide flux uncertainties. We use GENIE [22] to calculate the neutrino interaction cross sections and final state kinematics, in which the models for charged-current quasielastic scattering and scattering on a pair of correlated nucleons have been tuned based on data from T2K [23]. Neutrino interactions are simulated both inside the cryostat and outside where secondary products enter the detector. For both signal and background simulations, the decay or interaction products are propagated through a GEANT4 simulation of the detector. The response of the detector to both light and charge is simulated.

All three types of data (beam on, beam off, and simulated) are processed through the same chain of reconstruction algorithms. The optical reconstruction uses the PMT waveforms to produce "flashes" of coincident PMT hits. For the TPC information, we apply a twodimensional deconvolution of the signal waveforms on the wires within each plane [24,25]. Hits are formed from a Gaussian peak finding algorithm applied to the wire waveform. The Pandora framework [26] uses particle flow algorithms to cluster the hits of a single plane and, then, match clusters across planes into three-dimensional reconstructed objects, which Pandora classifies as "tracks" or "showers" based on a multivariate classifier score. Pandora also "slices" the event into groups of reconstructed objects that it considers to be independent interactions (either cosmogenic or beam induced) and removes well-identified cosmic slices. For any remaining slices, a flash-matching algorithm is applied to produce a PMT hit hypothesis using the reconstructed objects in the slice. The algorithm attempts to match the hypothetical PMT hit distribution with the observed flashes in the beam timing window, and calculates a $\chi^{2}$ value for the best match. The best matching slice of these remaining slices is labeled as the neutrino slice.

To preselect decay candidates in the event, we use the neutrino slices. The slice has to be matched to a PMT flash with a time of $[5.8,16.8] \mu$ s within the $20 \mu$ s PMT readout window (where the NuMI prompt neutrino spill produces flashes in the range $[6.1,15.7] \mu \mathrm{s})$, and the flash-matching $\chi^{2}$ has to be less than 10. An additional selection is imposed on the run 3 data, requiring that events cannot have a CRT hit in coincidence with the beam timing. The total number of objects in the slice has to be $\leq 5$, and of these, a maximum of 4 can be labeled as tracks. For all possible pairs of objects in the slice, the minimum distance between the object vertices (for reconstructed tracks, both start or end positions, and for showers, only start positions) is calculated. If this distance is less than $5 \mathrm{~cm}$, a decay vertex is produced at the midpoint between the object vertices with the minimum separation. The position of the decay vertex has to be reconstructed within the active volume of the detector. Slices with more than two objects could conceivably form multiple decay candidates, all of which are preselected and passed through the boosted decision tree (BDT) selection.

We apply two different BDTs to the preselected candidates: one trained against cosmic backgrounds and one trained against neutrino interactions simulated inside the cryostat. Each BDT is trained separately over the run 1 events and run 3 events, i.e., there are four BDTs in total. We split the run periods because the use of the CRT in run 3 and the differences between forward and reverse horn current operations can change the topologies and properties of the background distributions that the BDTs are trained against. We use XGBOOST [27] to train and apply the BDTs. We train the BDTs on ten input variables each. Nine of the ten input variables are the same for the cosmic-focused and neutrino-focused BDTs. These are (1) the opening angle between the two reconstructed objects; (2) the opening angle in the plane transverse to the hadron absorber direction from the detector center; $(3,4)$ the two angles between the two objects and the hadron absorber direction; (5) the Pandora track or shower score of the larger of the two objects (when ordered by number of hits); (6) the number of hits of the larger object; (7) the total number of hits contained in other objects in the slice, not including the two objects that form the decay candidate; (8) the maximum $y$ coordinate, relative to the decay vertex position, of shower start positions or track start or end positions, for any other objects in the slice; and (9) the minimum $z$ coordinate, relative to the decay vertex position, of shower start positions or track start or end positions, for any other objects in the slice. The last two variables are treated as "missing" within XGBOOST if the slice contains only two objects. The tenth input variable of the cosmic-focused BDT is the length of the larger object. The tenth input variable of the neutrino-focused BDT is the number of tracks in the slice. For all input variables and output BDT score distributions, we observe good data-simulation agreement in a control region of data with an early flash time (as the scalar boson signal is delayed by $\approx 600 \mathrm{~ns}$ with respect to the prompt neutrino interactions due to time-of-flight differences). 
The BDTs are trained on a signal simulation where each decay is of a scalar boson with $m_{S}$ uniformly chosen between 100 and $200 \mathrm{MeV} / c^{2}$, in order to reduce the dependence of the BDTs on $m_{S}$ in the range where $\theta_{\mathrm{KCV}}$ has not been excluded. The candidates used in the training have to be well reconstructed, with the cosmic contamination of each object below $10 \%$, and the reconstructed vertex and directions close to the generated values. The neutrino-focused BDT is trained against $10 \%$ of the simulated statistics of neutrino interactions in the cryostat, with the other $90 \%$ along with all the out-of-cryostat simulated interactions used for the sensitivity and limit calculations. Each reconstructed object used in the neutrino background training sample is required to have cosmic contamination below $10 \%$, similar to the signal sample. The cosmic-focused BDT is trained against beam-off candidates that fail the flash-matching $\chi^{2}$ requirement.

To select candidate decays, we require the two BDT scores to be above a minimum score. We choose the four minimum scores that maximize the sensitivity of the selection to the model parameter $\theta$ for $m_{S}=100 \mathrm{MeV} / c^{2}$, as we expect even better sensitivity at higher masses. The $95 \%$ confidence level (C.L.) sensitivity and limit are calculated for a single-bin counting experiment with the modified-frequentist CLs method, using the ROOSTATS statistical package [28], including systematic uncertainties as constrained Gaussian nuisance terms.

We consider several classes of systematic uncertainty. We include the simulation statistical uncertainty and beamoff data statistical uncertainty as uncertainties for the model prediction. The flux normalization uncertainty on the signal model is set to $30 \%$ as used by MiniBooNE [18]. The uncertainty on the background neutrino cross section modeling is evaluated by reweighting events using tools included with GENIE [29]. Interaction physics model parameters (both in GENIE and GEANT4) are varied multiple times within their 1 standard deviation uncertainties, and a weight is calculated for each simulated event between the central value and the modified model. The uncertainty on the event count in the selection is calculated from the standard deviation of weighted event counts across the variations. A similar procedure is followed to estimate the uncertainty on the background neutrino flux model due to hadron production uncertainties, using PPFX [19]. The flux uncertainty due to the beam line model (including focusing) is negligible compared to the hadron production uncertainty [15] and is not included.

Systematic uncertainties due to the modeling of the detector are evaluated through modified simulations varying parameters of the detector model. They are estimated to be $70 \%$ for the neutrino background simulation (dominated by the low statistics of simulated neutrino events in the
TABLE I. Systematic uncertainties for the signal and background model in the signal region.

\begin{tabular}{lcc}
\hline \hline Uncertainty & Background (\%) & Signal $(\%)$ \\
\hline Flux (hadron production) & 26.6 & 30.0 \\
Cross section model & 33.4 & not applicable \\
Detector model & 70.0 & 5.0 \\
Beam-off statistics & 38.0 & not applicable \\
Simulation statistics & 28.2 & $<2.0$ \\
\hline \hline
\end{tabular}

signal region after selection) and $5 \%$ for the signal simulation, taken to be the relative differences of event yields in the signal region between the central value and ten detector model variations summed in quadrature. The first five detector model variations are (1) uncertainties in the space charge mapping [30], (2) the ion-electron recombination model, (3) a decrease in light yield, (4) an increase in the Rayleigh scattering length, and (5) changing the light attenuation between the anode and cathode sides. We also modify the simulated TPC wire waveform amplitudes and widths. The sizes of the modifications are characterized in five dimensions based on hit positions, track angles with respect to the wires, and energy deposited per unit length. The modification sizes are estimated by comparing orthogonal data samples rich in protons and cosmic muons to the central value simulation. These five wire modifications (each dimension independently) are then applied to the signal and background simulation and used to extract the event yield variation. Although the uncertainty on the detector model for the background prediction is large, the final result is statistics-limited, and this uncertainty has minimal impact with respect to repeating the analysis with zero detector uncertainty. The uncertainties in the signal region after the optimal BDT selection are given in Table I.

After applying the BDT selection, the number of events expected for each background contribution and for several signal definitions are shown in Table II. The table also presents the estimated signal selection efficiency. The total

TABLE II. Estimated signal selection efficiency (eff.) for a scalar boson decay inside the TPC, and event yield [unweighted (unwt.) and beam-on exposure-weighted (exp. wt.), with the expected signal for $\left.\theta_{\mathrm{KCV}}\right]$.

\begin{tabular}{lcrc}
\hline \hline & & \multicolumn{2}{c}{ Event count } \\
\cline { 3 - 4 } Category & Eff. (\%) & Unwt. & Exp. Wt. \\
\hline Beam-off dataset & & 10 & $1.1 \pm 0.4$ \\
Neutrino simulation & & 16 & $0.8 \pm 0.7$ \\
Signal $\left(120 \mathrm{MeV} / c^{2}\right)$ & $14.0 \pm 0.8$ & 7268 & $4.9 \pm 1.5$ \\
Signal $\left(160 \mathrm{MeV} / c^{2}\right)$ & $14.9 \pm 0.9$ & 7654 & $12.2 \pm 3.6$ \\
\hline \hline
\end{tabular}




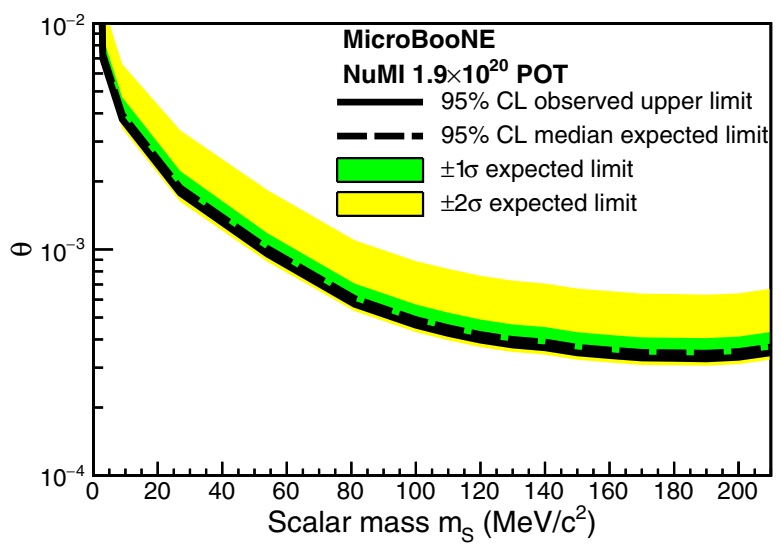

FIG. 2. The $95 \%$ confidence level sensitivity and observed limit of this search to the Higgs portal model parameter $\theta$.

expected background-only prediction is $1.9 \pm 0.8$ candidate events.

In the beam-on dataset, we observe two candidates in the signal region. We reject one candidate because its flash time of $5.84 \mu \mathrm{s}$ lies in the window between the start of the selection time $(5.8 \mu \mathrm{s})$ and the start of the neutrino interactions $(6.1 \mu \mathrm{s})$, making it an obvious cosmic background interaction. This post-selection cut only affects the cosmic background, reducing the cosmic acceptance by $2.7 \%$, with negligible effect on the sensitivity. When we manually inspect the TPC readout of the other candidate event, the two objects have the characteristics of a proton and a photon, and so, it is likely to be a neutrino-induced background.

With one observed event, we set the 95\% C.L. upper limit on the Higgs portal model presented in Fig. 2. The observed and expected limits for several scalar boson masses are enumerated in Table III and, for a wider range of masses, in the Supplemental Material [31]. The upper limit is compared with $\theta_{\mathrm{KCV}}$, along with other experimental limits, in Fig. 3. In Fig. 4, we present our result as a modelindependent limit on a new boson $X$ produced in $K^{+} \rightarrow \pi^{+} X$ decays, and decaying to $e^{+} e^{-}$pairs, for $X$ masses in the range $[100,210] \mathrm{MeV} / c^{2}$.

The limit presented in this publication rules out the remaining Higgs portal model parameter space required to

TABLE III. Observed (obs.) and expected [median (med.), \pm 1 , 2 standard deviation] $95 \%$ C.L. upper limits on the Higgs portal parameter $\theta$ for several scalar boson masses.

\begin{tabular}{|c|c|c|c|c|c|c|}
\hline \multirow{2}{*}{$\begin{array}{l}m_{S} \\
\left(\mathrm{MeV} / c^{2}\right)\end{array}$} & \multirow{2}{*}{$\begin{array}{c}\text { Obs. limit } \\
\left(10^{-4}\right)\end{array}$} & \multicolumn{5}{|c|}{ Expected limits $\left(10^{-4}\right)$} \\
\hline & & -2 s.d. & -1 s.d. & med. & +1 s.d. & +2 s.d. \\
\hline 120 & 4.0 & 3.7 & 3.9 & 4.2 & 4.9 & 7.6 \\
\hline 140 & 3.7 & 3.4 & 3.6 & 3.9 & 4.5 & 7.1 \\
\hline 160 & 3.4 & 3.2 & 3.3 & 3.6 & 4.2 & 6.5 \\
\hline
\end{tabular}

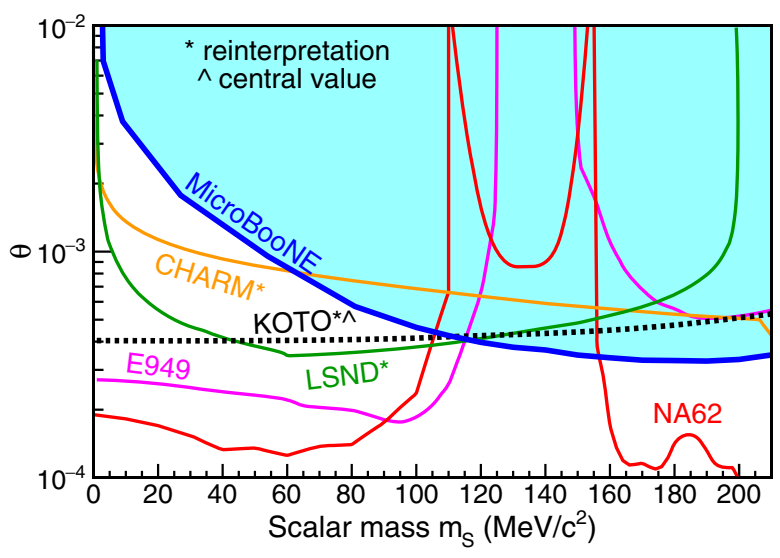

FIG. 3. The MicroBooNE 95\% C.L. upper limit (shaded) in the context of the model parameter $\theta_{\mathrm{KCV}}$ required to explain the central value of 1.78 counts in KOTO (dotted line), and exclusion contours from other experiments (solid lines; regions above the lines are excluded). The KOTO central value is adapted from Ref. [6] and scaled by $\sqrt{(3-1.22) / 3}$ to reflect updated background estimates from the KOTO collaboration [4]. The limits for E949 [8] and NA62 [9] are published by the collaborations, whereas the CHARM [6] and LSND [32] limits are reinterpretations of other searches.

explain the central value of a mild excess in KOTO at the 95\% confidence level. Our limit is the most constraining for $m_{S} \approx(120-160) \mathrm{MeV} / c^{2}$ and is directly derived from our own experimental data. The previous most stringent constraints in this range were reinterpretations of decadesold CHARM [6,33] and LSND [32] measurements, performed recently by independent authors without access to the raw experimental data. We have $\approx 2 \times 10^{21}$ POT of asyet unprocessed NuMI data along with $\approx 1 \times 10^{21}$ POT of currently blinded BNB data that we will analyze in the future and expect improved sensitivities [2].

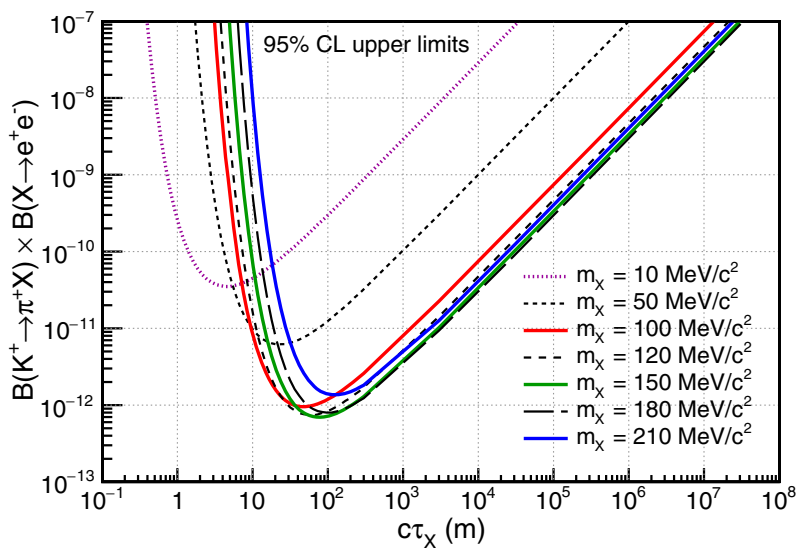

FIG. 4. Model-independent upper limits on the product of the production and decay branching ratios of a new boson $X$ as a function of the $X$ boson lifetime $\tau_{X}$ and mass $m_{X}$. 
This document was prepared by the MicroBooNE collaboration using the resources of the Fermi National Accelerator Laboratory (Fermilab), a U.S. Department of Energy, Office of Science, HEP User Facility. Fermilab is managed by Fermi Research Alliance, LLC (FRA), acting under Contract No. DE-AC02-07CH11359. MicroBooNE is supported by the following: the U.S. Department of Energy, Office of Science, Offices of High Energy Physics and Nuclear Physics; the U.S. National Science Foundation; the Swiss National Science Foundation; the Science and Technology Facilities Council (STFC), part of the United Kingdom Research and Innovation; and The Royal Society (United Kingdom). Additional support for the laser calibration system and cosmic ray tagger was provided by the Albert Einstein Center for Fundamental Physics, Bern, Switzerland. Participation of individual researchers in this project is supported by funds from the European Unions Horizon 2020 Research and Innovation Programme under the Marie SkłodowskaCurie Grant Agreement No. 752309. We thank Brian Batell, Joshua Berger, and Ahmed Ismail for outlining the search strategy.

*microboone_info@fnal.gov

[1] B. Patt and F. Wilczek, Higgs-field portal into hidden sectors, arXiv:hep-ph/0605188.

[2] B. Batell, J. Berger, and A. Ismail, Probing the Higgs portal at the Fermilab short-baseline neutrino experiments, Phys. Rev. D 100, 115039 (2019).

[3] S. Shinohara (KOTO Collaboration), Search for the rare decay $K_{L} \rightarrow \pi^{0} \nu \bar{\nu}$ at J-PARC KOTO experiment, J. Phys. Conf. Ser. 1526, 012002 (2020).

[4] J. K. Ahn et al. (KOTO Collaboration), Study of the $K_{L} \rightarrow$ $\pi^{0} \nu \bar{\nu}$ Decay at the J-PARC KOTO Experiment, Phys. Rev. Lett. 126, 121801 (2021).

[5] T. Kitahara, T. Okui, G. Perez, Y. Soreq, and K. Tobioka, New Physics Implications of Recent Search for $K_{L} \rightarrow \pi^{0} \nu \bar{\nu}$ at KOTO, Phys. Rev. Lett. 124, 071801 (2020).

[6] D. Egana-Ugrinovic, S. Homiller, and P. Meade, Light Scalars and the Koto Anomaly, Phys. Rev. Lett. 124, 191801 (2020).

[7] P. S. Bhupal Dev, R. N. Mohapatra, and Y. Zhang, Constraints on long-lived light scalars with flavor-changing couplings and the KOTO anomaly, Phys. Rev. D 101, 075014 (2020).

[8] A. V. Artamonov et al. (E949 Collaboration), Study of the decay $K^{+} \rightarrow \pi^{+} \nu \bar{\nu}$ in the momentum region $140<P_{\pi}<199 \mathrm{MeV} / c$, Phys. Rev. D 79, 092004 (2009).

[9] E. Cortina Gil et al. (NA62 Collaboration), Measurement of the very rare $K^{+} \rightarrow \pi^{+} \nu \bar{\nu}$ decay, J. High Energy Phys. 06 (2021) 093.

[10] P. Abratenko et al. (MicroBooNE Collaboration), Search for heavy neutral leptons decaying into muon-pion pairs in the MicroBooNE detector, Phys. Rev. D 101, 052001 (2020).

[11] I. Stancu et al., Technical design report for the $8 \mathrm{GeV}$ beam, Technical Report No. FERMILAB-DESIGN-2001-03,
Fermi National Accelerator Lab. (FNAL), Batavia, 2001, https://doi.org/10.2172/1212167.

[12] R. Acciarri et al. (MicroBooNE Collaboration), Design and construction of the MicroBooNE detector, J. Instrum. 12, P02017 (2017).

[13] C. Adams et al. (MicroBooNE Collaboration), Design and construction of the MicroBooNE Cosmic Ray Tagger system, J. Instrum. 14, P04004 (2019).

[14] P. Adamson et al., The NuMI neutrino beam, Nucl. Instrum. Methods Phys. Res., Sect. A 806, 279 (2016).

[15] P. Abratenko et al. (MicroBooNE Collaboration), Measurement of the flux-averaged inclusive charged-current electron neutrino and antineutrino cross section on argon using the NuMI beam and the MicroBooNE detector, Phys. Rev. D 104, 052002 (2021).

[16] L. Aliaga et al. (MINER $\nu$ A Collaboration), Neutrino flux predictions for the NuMI beam, Phys. Rev. D 94, 092005 (2016); Phys. Rev. D 95, 039903(A) (2017).

[17] S. Agostinelli et al. (GEANT4 Collaboration), Geant4-a simulation toolkit, Nucl. Instrum. Methods Phys. Res., Sect. A 506, 250 (2003).

[18] A. A. Aguilar-Arevalo et al. (MiniBooNE Collaboration), First Measurement of Monoenergetic Muon Neutrino Charged Current Interactions, Phys. Rev. Lett. 120, 141802 (2018).

[19] L. A. Soplin, Neutrino flux prediction for the NuMI beamline, Ph.D. thesis, William-Mary Coll., 2016.

[20] C. E. Patrick et al. (MINER $\nu$ A Collaboration), Measurement of the muon antineutrino double-differential cross section for quasielastic-like scattering on hydrocarbon at $E_{\nu} \sim 3.5 \mathrm{GeV}$, Phys. Rev. D 97, 052002 (2018).

[21] M. A. Acero et al. (NOvA Collaboration), New constraints on oscillation parameters from $\nu_{e}$ appearance and $\nu_{\mu}$ disappearance in the NOvA experiment, Phys. Rev. D 98, 032012 (2018).

[22] C. Andreopoulos et al., The GENIE neutrino Monte Carlo generator, Nucl. Instrum. Methods Phys. Res., Sect. A 614, 87 (2010).

[23] K. Abe et al. (T2K Collaboration), Measurement of doubledifferential muon neutrino charged-current interactions on $\mathrm{C}_{8} \mathrm{H}_{8}$ without pions in the final state using the T2K off-axis beam, Phys. Rev. D 93, 112012 (2016).

[24] C. Adams et al. (MicroBooNE Collaboration), Ionization electron signal processing in single phase LArTPCs. Part I. Algorithm Description and quantitative evaluation with MicroBooNE simulation, J. Instrum. 13, P07006 (2018).

[25] C. Adams et al. (MicroBooNE Collaboration), Ionization electron signal processing in single phase LArTPCs. Part II. Data/simulation comparison and performance in MicroBooNE, J. Instrum. 13, P07007 (2018).

[26] R. Acciarri et al. (MicroBooNE Collaboration), The Pandora multi-algorithm approach to automated pattern recognition of cosmic-ray muon and neutrino events in the MicroBooNE detector, Eur. Phys. J. C 78, 82 (2018).

[27] T. Chen and C. Guestrin, XGBoost: A scalable tree boosting system, in Proceedings of the 22nd ACM SIGKDD International Conference on Knowledge Discovery and Data Mining, KDD '16 (ACM, New York, NY, 2016), pp. 785-794. 
[28] L. Moneta, K. Belasco, K. S. Cranmer, S. Kreiss, A. Lazzaro, D. Piparo, G. Schott, W. Verkerke, and M. Wolf, The RooStats project, Proc. Sci. ACAT2010 (2010) 057.

[29] C. Andreopoulos, C. Barry, S. Dytman, H. Gallagher, T. Golan, R. Hatcher, G. Perdue, and J. Yarba, The GENIE neutrino Monte Carlo generator: Physics and user manual, arXiv:1510.05494.

[30] P. Abratenko et al. (MicroBooNE Collaboration), Measurement of space charge effects in the MicroBooNE LArTPC using cosmic muons, J. Instrum. 15, P12037 (2020).
[31] See Supplemental Material at http://link.aps.org/ supplemental/10.1103/PhysRevLett.127.151803 for tabulated values of the signal selection efficiency and the observed and expected upper limits on $\theta$.

[32] S. Foroughi-Abari and A. Ritz, LSND constraints on the Higgs portal, Phys. Rev. D 102, 035015 (2020).

[33] F. Bergsma et al. (CHARM Collaboration), Search for axion-like particle production in $400 \mathrm{GeV}$ proton-copper interactions, Phys. Lett. 157B, 458 (1985). 